\title{
Toward a simulation-based approach for emergency evacuation route planning in metro stations
}

\author{
Xianguo WU ${ }^{1}$, Mengjie $\mathrm{LIU}^{1}$, Limao $\mathrm{ZHANG}^{1,2,3}$ \\ 1. Civil Engineering \& Mechanics, Huazhong University of Science and Technology, China \\ 2. Department of Civil and Environmental Engineering, University of Alberta, Canada \\ 3. Corresponding author's e-mail:limao@ualberta.ca
}

\begin{abstract}
This paper presents a systematic simulation-based approach with detailed step-by-step procedures for route planning in emergency evacuation in metro stations. In accordance with emergency evacuation mechanism analysis, the length of evacuation route (L), the time of evacuation $(\mathrm{T})$ and the density of pedestrian flow (D) are identified as critical factors that affect the performance of emergency evacuation. With all the critical factors, such as L, T and D, taken into account, a comprehensive multi-attribute decision algorithm is developed in order to optimize the selection of evacuation route under emergency. Taking the Hongshan Station in Wuhan metro systems as an example, the Anylogic tool is used to simulate the scenario of evacuation route planning in case of a fire. The simulation results regarding the evacuation performance indicators are analyzed and compared between the traditional and proposed approaches. Results indicate that the proposed approach can reduce the evacuation time without increasing the route length, and improve greatly the crowded conditions of pedestrian flow. The developed approach can provide guidelines and support for the optimization of evacuation route planning under emergency conditions.
\end{abstract}

\section{KEYWORDS}

simulation; emergence evacuation; route planning; case study; metro station

\section{INTRODUCTION}

Subway construction, as an important part of urban rail transit construction, is an important guarantee for economic development and social progress, at the same time, as a convenient, safe and reliable means of transport, subway is more and more widely favored in the contemporary city. However, with the increase of the passengers, there are series of problems to be solved in subway operation. Issues such as passenger congestion, fire evacuation, operating equipment failure became the focus of operations management, once catastrophes occur, the personnel and economic losses would be quite serious.

At present, subway operations management has received considerable attention in the past and is becoming increasing important. Subway operator safety evaluation system has been established, researches for evacuation of subway station mainly focused on the density of the flow at the key points, capacity of devices which will reduce the efficiency of evacuation, like walkways and stairs are studied, as well as the service efficiency of security equipment and ticketing facilities. In traditional evacuation route planning, only the length of the evacuation route is considered, or only focus on evacuation time. However, the evacuation route planning is affected by many factors, not 
simply pursuing of the shortest distance or minimum time. We can see that, length based model may cause overcrowding and delay the evacuation, time based model may increase the length of evacuation or cause congestion, all these cases have great psychological impact on passengers. Therefore, the length of evacuation, the density of pedestrian flow in key points, the evacuation time and other factors are considered in the study, an emergency evacuation route planning is made. The Anylogic tool is used to simulate the scenario of evacuation route planning in case of a fire, the feasibility of proposed method is proved in this simulation. It is demonstrated that this model provides a reference for decision-making in emergency evacuation of metro station.

\section{METHOD}

The evacuation route planning can be affected by both the layout and the number of selected, which will cause crowding and reduce evacuation efficiency. Therefore, pedestrian flow plays a great role in evacuation, which can signify the pedestrian velocity and the flow of evacuation process. By building the evacuation network, the optional routes in emergency evacuation is confirmed. The evacuation route planning can be made in consideration of the length of evacuation route (L), the time of evacuation (T) and the density of pedestrian flow (D).

The length of evacuation route is confirmed as in Eq. (1). L is scored as Eq. (2).

$$
\begin{aligned}
& L_{r}=\sum_{k=1}^{m} a_{k} \cdot w_{k}, \quad(r=1,2 \cdots \mathrm{p}) \\
& l_{r}=L_{r} / 60
\end{aligned}
$$

Time of evacuation is defined as Eq. (3). L is scored as Eq. (4).

$$
\begin{aligned}
& \mathrm{T}_{r}=\sum_{k=1}^{m} T_{k}+\sum_{c=1}^{v} t_{c}, \quad(r=1,2 \cdots \mathrm{p}) \\
& t_{r}=T_{r} / 100
\end{aligned}
$$

Density of pedestrian flow (D) is calculated as Eq. (5). The density range and score is given by the level of risk, as shown in Table 1. D is scored as Eq. (6).

$$
\begin{aligned}
& D=Q / A \\
& \rho_{r}=\sum \rho_{j}+(Y-y) * 1.0
\end{aligned}
$$

Table 1. The density range and score graded by the level of risk

\begin{tabular}{llll}
\hline \multirow{2}{*}{ Risk level } & \multicolumn{2}{l}{ density $\left(\mathrm{p} / \mathrm{m}^{2}\right)$} & \multirow{2}{*}{ Score $\rho$} \\
\cline { 2 - 3 } & In hall & In stairs & 0.2 \\
I & $>2.40$ & $>3.75$ & 0.4 \\
II & $2.08 \sim 2.40$ & $3.25 \sim 3.75$ & 0.6 \\
III & $1.92 \sim 2.08$ & $3 \sim 3.25$ & 0.8 \\
IV & $1.76 \sim 1.92$ & $2.75 \sim 3$ & 1.0 \\
No & $<1.76$ & $<2.75$ & \\
\hline
\end{tabular}


In traditional evacuation route planning, the probability that passenger $\mathrm{m}$ select route $\mathrm{r}$ is shown in Eq. (7). Considering three indexes, namely, the length of evacuation route ${ }^{l_{r}}$, the time of evacuation

$t_{r}$ and the density of pedestrian flow $\rho_{r}$, the probability that passenger $\mathrm{m}$ select route $\mathrm{r}$ is shown in Eq. (8).

$$
\begin{aligned}
& \operatorname{prob}_{m r}=\frac{1}{l_{r}^{a} f_{r}^{b}} \\
& Z_{r}=\frac{\rho_{r}^{\gamma}}{l_{r}^{\alpha} t_{r}^{\beta}}
\end{aligned}
$$

Based on these theories, the flowchart of evacuation route planning of emergency evacuation in subway station is shown in Figure 1, the details is shown step by step as follows:

1) The evacuation network is established in consideration of evacuation theory and practical situation.

2) The length of the $\mathrm{N}$ shortest evacuation routes are confirmed with Dijkstra algorithm.

3) Considered the layout of station, ${ }_{r}$, the length of evacuation route, is confirmed, as shown in Eq.

(1). Then $l_{r}$ is calculated with continuous interpolation method, as shown in Eq. (2).

4) $\mathrm{T}_{r}$, the time of evacuation, is simulated with Anylogic, the math is given in Eq. (3). Then, $t_{r}$ is calculated as shown in Eq. (4), where 100s is set as the standard evacuation time.

5) Based on the evacuation network, the key points that affect evacuation efficiency are found. Density of pedestrian flow (D) is calculated with Eq. (5), according to the traditional risk grading strategy, $\rho_{r}$ is confirmed as shown in Table 1.

6) $Z_{r}$, the score of route $r$, is got with method that synthesized by additive synthesis method and multiplicative synthesis method, as shown in Eq. (8), the route with the highest score is the optimal route. Step 3,4,5,6 is used to get $Z_{r}$ until all people left. 


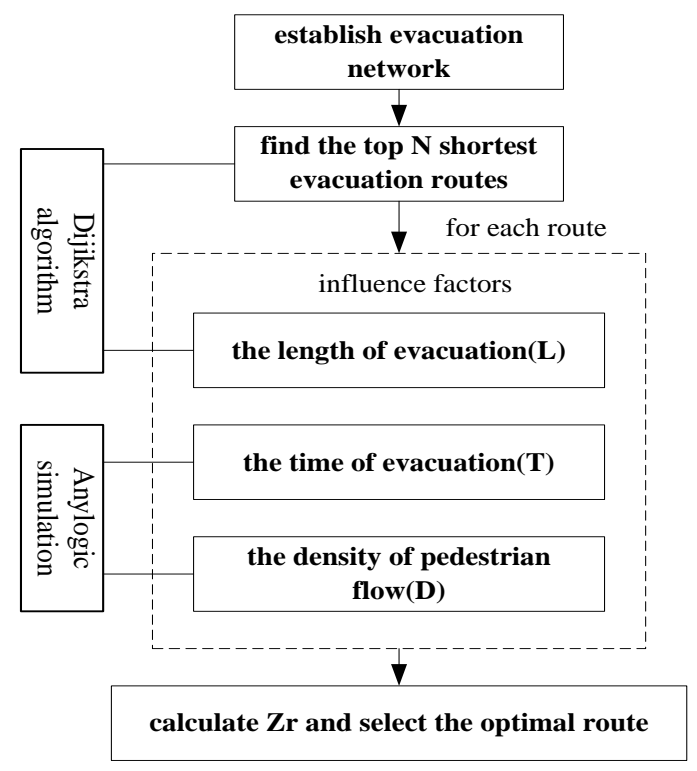

Figure 1. Flowchart of evacuation route planning of emergency evacuation in subway station

\section{RESULTS AND DISCUSSION}

The simulation network is established as Figure 2. Where, 1 11 are evacuation notes, $N=\{1,2, \cdots, 10,11\}$, specifically, 4 and 9 is the sites of turnstiles, 1, 2, 3 and 8 are channels connecting with the platform, 5, 6, 7, 10 and 11 are exits. While a1-a10 are connections between notes, $A=\{a 1, a 2 \cdots, a 10\}$. Moreover, in evacuation, passengers in the subway hall tend to select shorter route, while passengers in the platform select route by experience and crowding, namely the length of evacuation has more influence at connections between turnstiles and exits. Hence, the weights of connections between turnstiles and exits are 1 , but the weights of connections between turnstiles and channels are 0.5 , the set of weights is $W=\{0.5,0.5,0.5,1,1,1,0.5,1,1,1\}$. The points marked with red are the key points in evacuation, where density of pedestrian flow are required to measure and control. On these basis, 12 evacuation routes, I-XII, are established in the Hongshan station, as given in Table 2 .

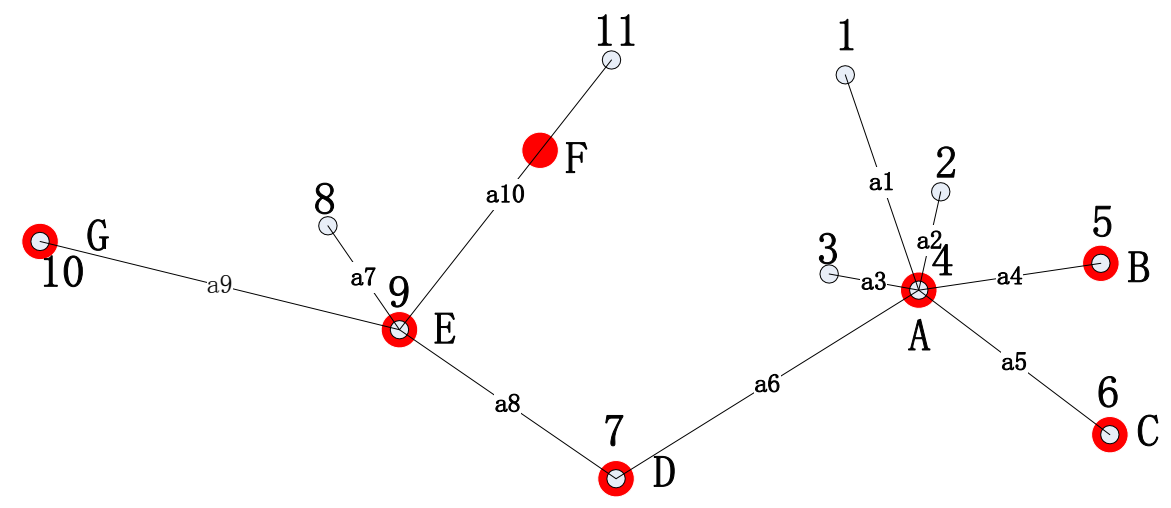

Figure 2 The evacuation network of the Hongshan station 
Table 2. The evacuation routes of the Hongshan station

\begin{tabular}{ccccc}
\hline Number & Evacuation route & Weight & Length & Key points \\
\hline I & $1-4-5$ & $0.5,1$ & 42 & \\
II & $2-4-5$ & $0.5,1$ & 38.5 & A、B \\
III & $3-4-5$ & $0.5,1$ & 33.5 & \\
IV & $1-4-6$ & $0.5,1$ & 55.5 & A、C \\
V & $2-4-6$ & $0.5,1$ & 52 & \\
VI & $3-4-6$ & $0.5,1$ & 47 & A、D \\
VII & $1-4-7$ & $0.5,1$ & 67.5 & \\
VIII & $2-4-7$ & $0.5,1$ & 64 & E、D \\
IX & $3-4-7$ & $0.5,1$ & 59 & E、G \\
X & $8-9-7$ & $0.5,1$ & 50.5 & E、F \\
XI & $8-9-10$ & $0.5,1$ & 64.5 & 84 \\
XII & $8-9-11$ & $0.5,1$ &
\end{tabular}

Based on the model, the traffic density distribution map is shown in Figure 3. it can be seen that, the preliminary simulation is coincided with the assumption that crowding are mainly found in turnstiles and exits. It also shows that the simulation model is feasible.

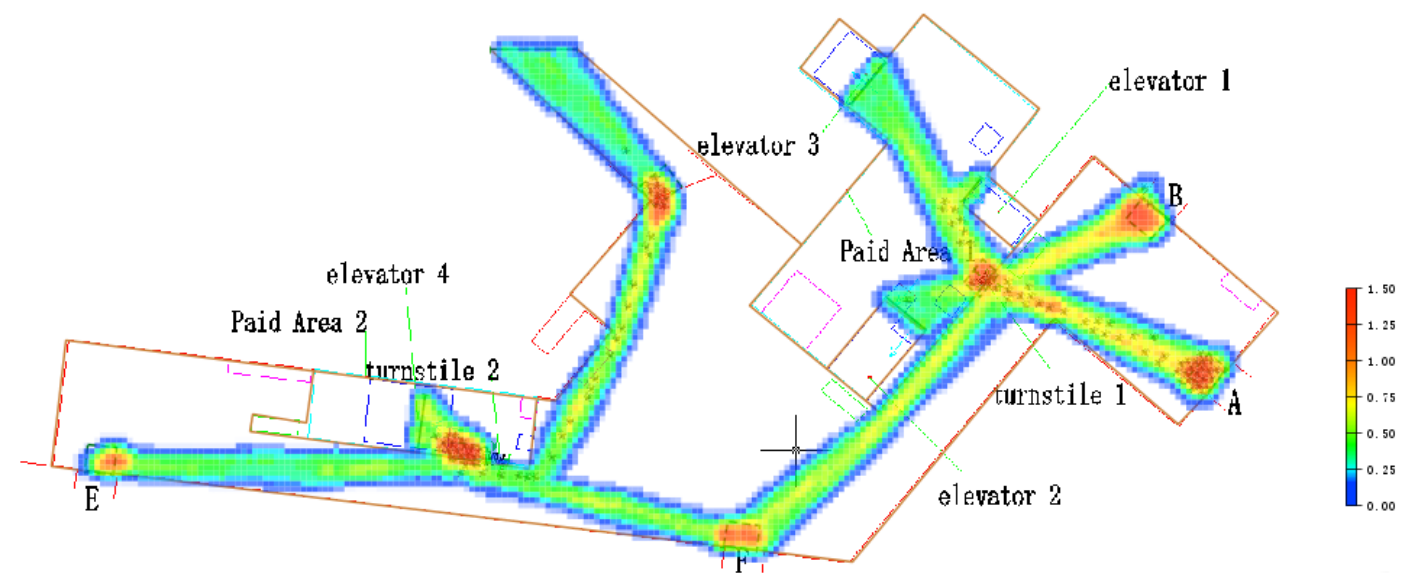

Figure 3 The traffic density distribution map

The density of pedestrian flow in key points are accounted, the line chart is shown in Figure 4. At the same time, the other simulation is carried out based on traditional method that only the length of route is considered, the result is shown in Figure 5. In addition, after statistical analysis of the length of evacuation route and the evacuation time of the 50 times simulation, the mean and standard deviation is summarized in Figure 6 and Figure 7. 


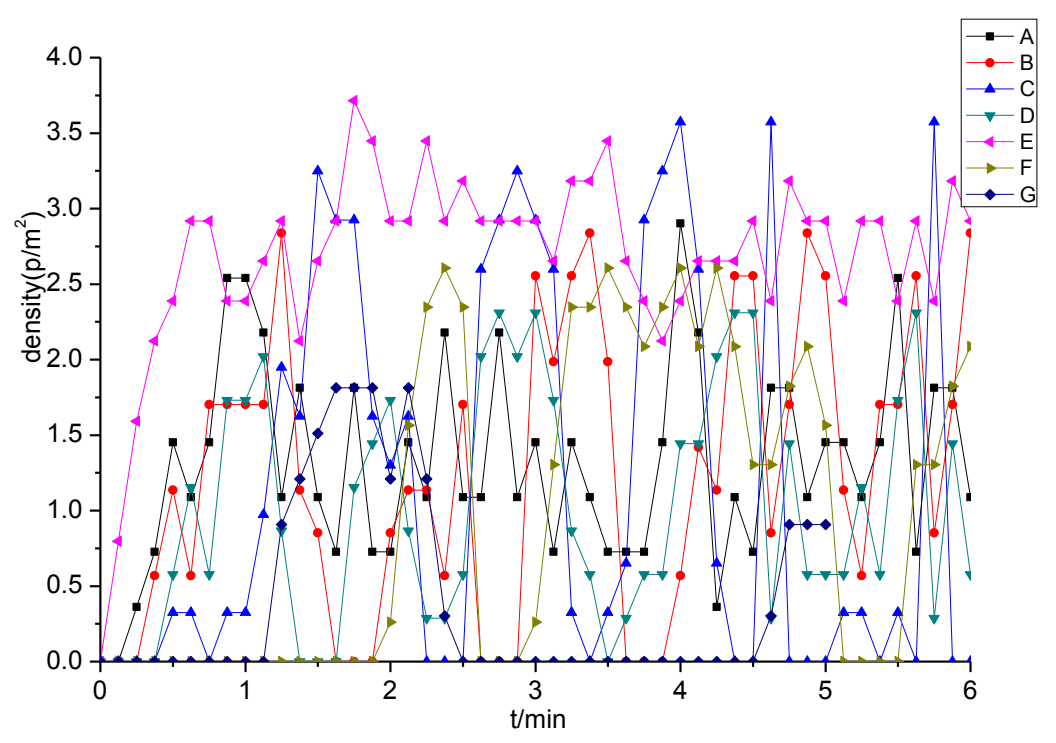

Figure 4 The density of pedestrian flow in key points of multi-attribute decision-making model

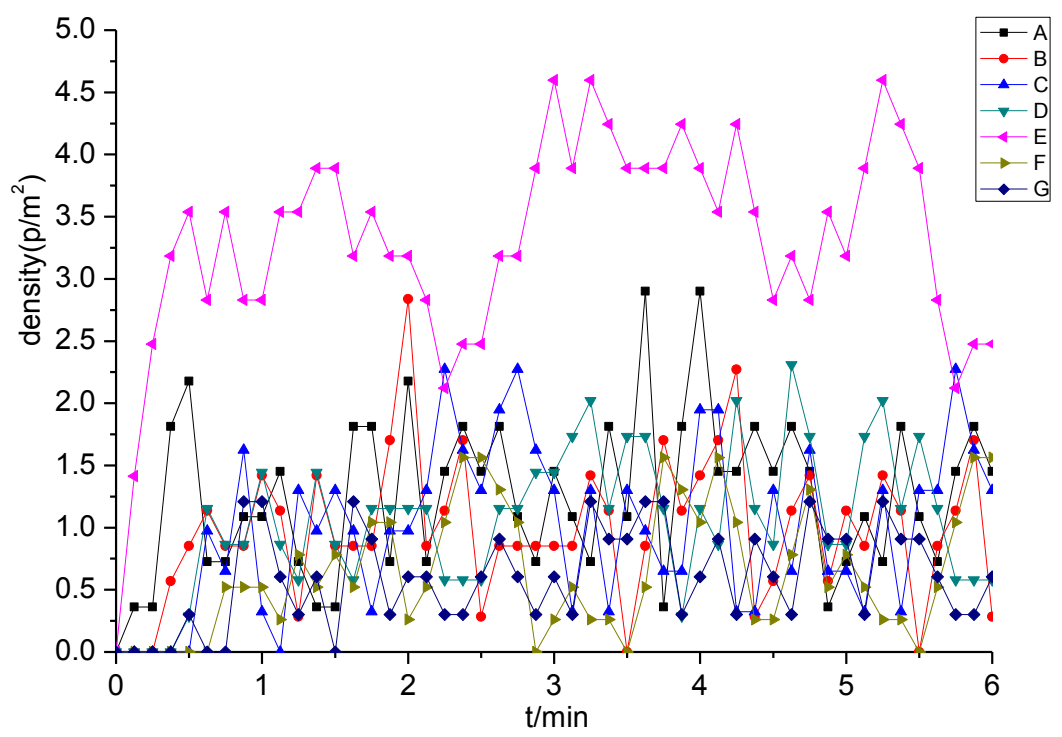

Figure 5 The density of pedestrian flow in key points of traditional method

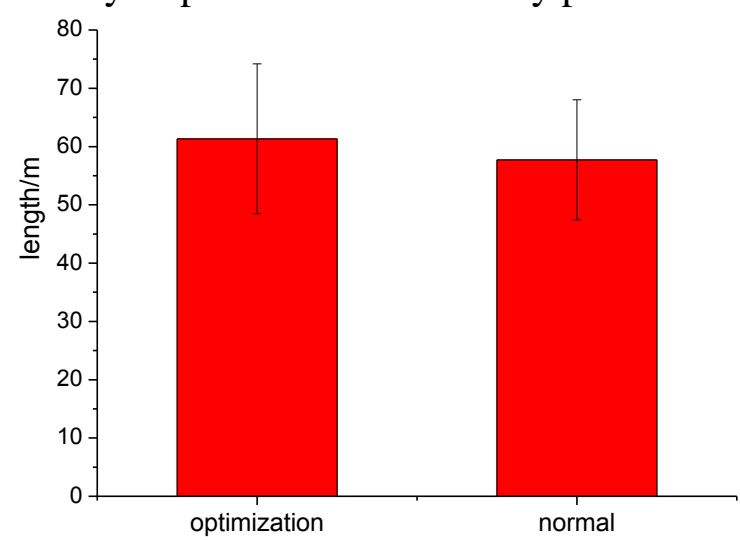

Figure 6 The mean and standard deviation of the length of evacuation route 


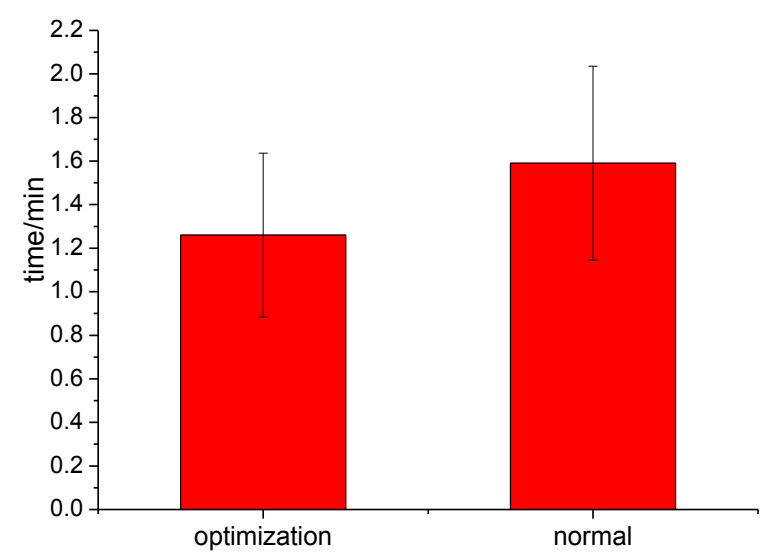

Figure 7 The mean and standard deviation of the evacuation time

Compering the multi-attribute decision-making method and traditional length based method, analyzing from the length of evacuation, the density of pedestrian flow, and the evacuation time, the simulation results is summarized in Table 3.

Table 3. Simulation results of evacuation route planning

\begin{tabular}{|c|c|c|c|c|c|c|c|c|c|}
\hline \multirow{2}{*}{ Simulation model } & \multirow{2}{*}{$\begin{array}{c}\text { Average walking } \\
\text { length } / \mathrm{m}\end{array}$} & \multicolumn{7}{|c|}{ Average density of pedestrian flow $/\left(\mathrm{p} / \mathrm{m}^{2}\right)$} & \multirow{2}{*}{$\begin{array}{c}\text { Average evacuation } \\
\text { length } / \mathrm{min}\end{array}$} \\
\hline & & A & $B$ & $\mathrm{C}$ & $\mathrm{D}$ & $\mathrm{E}$ & $\mathrm{F}$ & G & \\
\hline $\begin{array}{l}\text { multi-attribute } \\
\text { decision-making }\end{array}$ & 61.32 & 2.35 & 1.21 & 1.65 & 1.56 & 3.06 & 0.82 & 0.87 & 1.26 \\
\hline length based & 57.72 & 3.85 & 1.93 & 3.12 & 1.33 & 4.14 & 1.27 & 1.78 & 1.59 \\
\hline
\end{tabular}

From these figures, it can be seen that the difference of the average walking length between the multi-attribute decision-making model and traditional model is small. But the modified model is obviously superior to the traditional model in the length of evacuation and the density parameter. By analyzing Figure 4 and Figure 5 we can find that, relative to Figure 5, the curves in Figure 4 are volatile but periodic, it means although crowding is existed in this method, it does not last long, so the average is significantly reduced. Generally, brief crowded will not cause confusion, therefore this method is favorable for emergency evacuation, the conclusion can be testified in Table 3. Comparing the evacuation time, the average value in multi-attribute decision-making model is $1.26 \mathrm{~min}$, while it is $1.59 \mathrm{~min}$ in length based model, about $20 \mathrm{~s}$ is reduced, the optimization is highly significant. In conclusion, the multi-attribute decision-making model is very applicable to the evacuation route planning in urban transit traffic.

\section{CONCLUSION}

Based on the traditional length based or time based route planning method, three factors are selected to determine the optimal route. The Hongshan Station in Wuhan metro systems is taken as an example. The Anylogic tool is used to simulate the scenario of evacuation route planning in case of a fire. The results verify the proposed model through different scenarios. The contribution of this study can be summarized as follows.

(1) To meet the needs of saving time and psychological demands for passengers in evacuation, in accordance with emergency evacuation mechanism analysis, the length of evacuation route (L), the 
time of evacuation (T) and the density of pedestrian flow (D) are identified as critical factors that affect the performance of emergency evacuation.

(2) In combination with criterion and other requirements, the quantitative methods for three determinants are proposed in this study. In addition, reference to existed study, method that synthesized by additive synthesis method and multiplicative synthesis method is used in the study,

the final score is confirmed by $Z_{r}=\rho_{r}^{\gamma} /\left(l_{r}^{\alpha} t_{r}^{\beta}\right)$, it is the mainly foundation of the evacuation route planning.

(3) The evacuation efficiency of optimization method is proved by the simulation of the Hongshan Station. According to the structure and function layout, in accordance with investigation result, the simulation environment is set. Then, the simulation model is established on simulation tool Anylogic. The simulation result is demonstrated through three scenarios, average walking length, average densities of key points, and average evacuation time. Comparing with traditional single length based or time based method, this proposed model is more favorable in emergency evacuation of metro station.

\section{REFERENCES}

Dai, Baoqian, Wang, Tong, Jiang, Yukun, et al. (2006). Study on Index System of Comprehensive Safety Assessment for Subway Operation System. China Safety Science Journal, 12, 9-14.

He Jianfei, Liu Xiao (2013). A Subway Emergency Evacuation Optimization Method Based On Congestion Degree. China Safety Science Journal, 2 166-171.

Hu Pengfei(2013). The Research of Pedestrian Flow Abnormity State Identification and Pre-warning System Design in Comprehensive Passenger Transport Terminal, Jilin University.

Jia Hongfei, Yang Lili, Tang Ming (2009). Pedestrian Flow Characteristics Analysis and Model Parameter Calibration in Comprehensive Transport Terminal. Journal of Transportation Systems Engineering and Information Technology, 5 , 117-123.

Jiang Yangsheng, Li Xi, An Ding, et al. (2014). Simulation optimization of ticket vending machines' configuration in metro station considering randomness and level of service. JOURNAL OF SOUTHEAST UNIVERSITY ( Natural Science Edition), 2, 430-435.

Li Qingquan, Li Qiuping, Fang Zhixiang (2011). An Emergency Evacuation Routing Optimization Method Based on Space-time Congestion Concept. Acta Geodaetica et Cartographica Sinica, 4, 517-523.

Liu Wenting (2008). Emergency Evacuation Capacity of Pedestrian in Urban Rail Transit Station. School of Transportation Engineering.

Zhang Yunming (2011). A quantitative study of the original parameter of evacuation. Fire Science and Technology, 6 , 496-499.

Zheng X, Zhong T, Liu M. (2009). Modeling crowd evacuation of a building based on seven methodological approaches. Building and Environment. 44(3), 437-445. 